\title{
52 Endurance training and the capillaries
}

The key benefits of physical activity and exercise are:

- Capillary neogenesis

- Opening of resting capillaries

- Formation of collateral circulation

- Maintaining elasticity

- Widening of the lumen

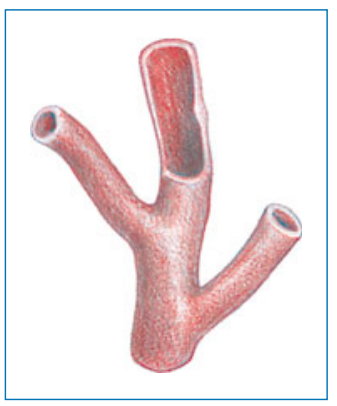

- Fig. $\mathbf{5 2 . 1}$

Neogenesis of capillaries occurs specifically in the working muscles. This improves the oxidative capacity given that it increases the volume of the mitochondria at the same time. Mitochondria are the cells' power houses where energy is generated via the citrate cycle and the respiratory chain ( $\triangleright$ Chapter 6 ). The cell's metabolism becomes more effective in that less oxygen is consumed to achieve the same output.

To wit, the blood pressure elevation during physical activity increases capillary density, dilates resting capillaries and leads to the formation of a collateral circulation ("watershed effect"). Blood volume is more effectively distributed and energy is generated by the citric acid cycle and respiratory

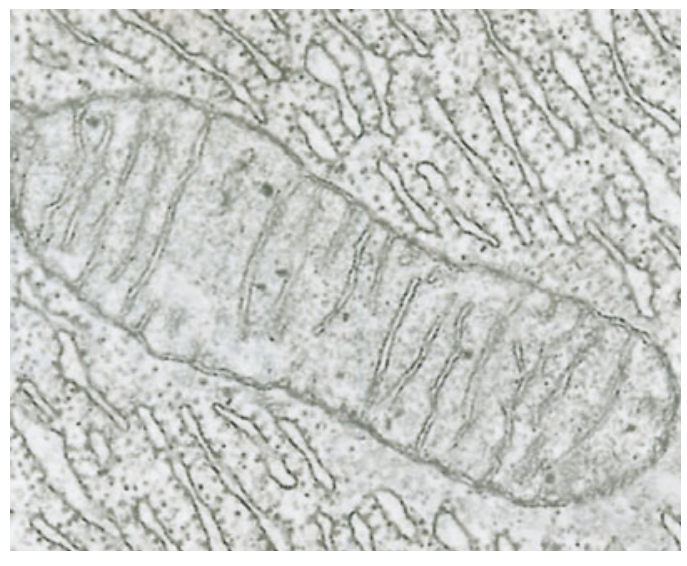

- Fig. 52.2 Electron microscopic recording of a mitochondrion

chain ( $\downarrow$ Chapter 6). Cell metabolism becomes more effective by reducing the oxygen demand for the same level of exertion.

The higher blood pressure during physical activity produces an increase in capillary density and resting oxygen supply is better utilized. The vessels remain more elastic. An exercise regimen of more than 5 hours per week widens the blood vessel lumen, significantly increases localized blood flow and markedly lowers the resting blood pressure $(\triangleright$ also Chapter 53). 